\title{
DISORDERED Nd-DOPED CRYSTALS FOR DIODE PUMPING
}

\author{
W. RYBA-ROMANOWSKI \\ Institute for Low Temperature and Structure Research, Polish Academy of Sciences \\ Okólna 2, 50-950 Wrocław, Poland
}

\begin{abstract}
Some interest has been directed recently to neodymium doped crystals with strong inhomogeneous broadening of absorption bands which are believed to be better suited for diode pumping than the crystals with ordered lattice. The disordered Nd-doped crystals may be obtained in two ways. The first one is to introduce a structural disorder in ordered lattice by substituting partially one or more cations by cations with different charges. Alternatively, the compounds with inherently disordered structure are synthesized and doped with $\mathrm{Nd}$. We consider two wide families of compounds of general formulae $\mathrm{ABC}_{3} \mathrm{O}_{7}$ and $\mathrm{ABCO}_{4}$ where $\mathrm{A}$ stands for $\mathrm{Ca}, \mathrm{Sr}, \mathrm{Ba}, \mathrm{B}$ is $\mathrm{Y}$ or rare earth and $\mathrm{C}$ stands for $\mathrm{Ga}$ or $\mathrm{Al}$. Both the families form the crystals with inherently disordered structure and can accept an appreciable amount of $\mathrm{Nd}$. Results of structural and spectroscopic investigations are used to determine material's parameters relevant to laser performance. Comparison of several disordered crystals is made and their suitability for diode pumping is discussed.
\end{abstract}

PACS numbers: $78.45 .+\mathrm{h}, 78.55 . \mathrm{Hx}$

In the past few years a considerable progress has been made in the field of laser diodes. Powerful laser diodes are now easily available and they are replacing the conventional broad band sources of optical pumping for rare earth doped laser materials. In particular, the emission of the AlGaAs laser diode matches perfectly the absorption band of neodymium situated at about $800 \mathrm{~nm}$ and corresponding to the ${ }^{4} I_{9 / 2} \rightarrow{ }^{4} F_{5 / 2},{ }^{2} H_{9 / 2}$ transition.

The energy level scheme for trivalent neodymium in dielectric crystals is shown in Fig. 1. It can be seen that the pump level and the upper laser level are separated by small energy gap of about $2000 \mathrm{~cm}^{-1}$, thus the excitation decays quickly by multiphonon emission to upper laser level. Since the wavelength of laser transition is close to $1.06 \mu \mathrm{m}$ and the wavelength of the pump transition is about $808 \mathrm{~nm}$, the energy loss due to photon decrement is only $24 \%$. As a result, the heating of laser active material is considerably less important than in the case of broad band pumping to higher lying levels. There are however some characteristic properties of laser diode which should be taken into account. First, the spectral bandwidth of laser diode emission is relatively broad. Second, the wavelength of laser diode emission depends strongly on diode temperature, typically 


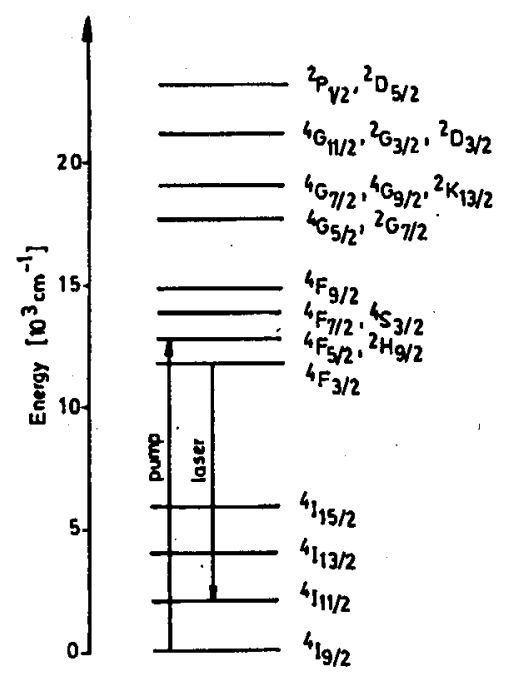

Fig. 1. Energy level scheme for $\mathrm{Nd}^{3+}$ in dielectric crystals.

$0.2-0.3 \mathrm{~nm} \mathrm{deg}{ }^{-1}$. These properties may influence the performance of a diode pumped solid state neodymium laser.

In the upper part of Fig. 2 the emission band of a commercial AlGaAs laser diode is shown. Below there is an absorption band of neodymium doped yttrium aluminum garnet (YAG:Nd), the crystal widely used as a laser material. Two vertical lines in the figure indicate positions of maxima of laser diode emission at $18^{\circ} \mathrm{C}$ and $30^{\circ} \mathrm{C}$ and these values are real ambient temperatures. It has been shown experimentally that the change of temperature within these limits results in significant variation of laser output power in the case of yttrium aluminum garnet [1]. Of course, the laser diode temperature may be and actually is controlled but thermocoolers are highly expensive and power consuming devices. The overall cost of a diode pumped laser is therefore high and its efficiency is reduced. That is why some interest has been directed recently to laser active materials characterized by strong inhomogeneous broadening of absorption bands. An example of such a band is shown in the bottom of Fig. 2. In this case a temperature control is less stringent, thus the wavelength shift due to ageing of a diode laser will affect laser performance in more moderate manner.

Neodymium doped laser glasses would be materials of choice but they have important disadvantage, namely the poor thermal conductivity, which limits seriously the laser power levels. Thus, the group of materials called disordered crystals are considered. The notion "disordered crystals" is used to denote crystalline materials characterized by certain structural disorder which results in strong inhomogeneous broadening of spectral bands corresponding to electronic transitions of rare earth ions. Essentially, disordered crystals may be obtained in two ways. The first one is to induce a structural disorder in ordered lattice by substituting par- 


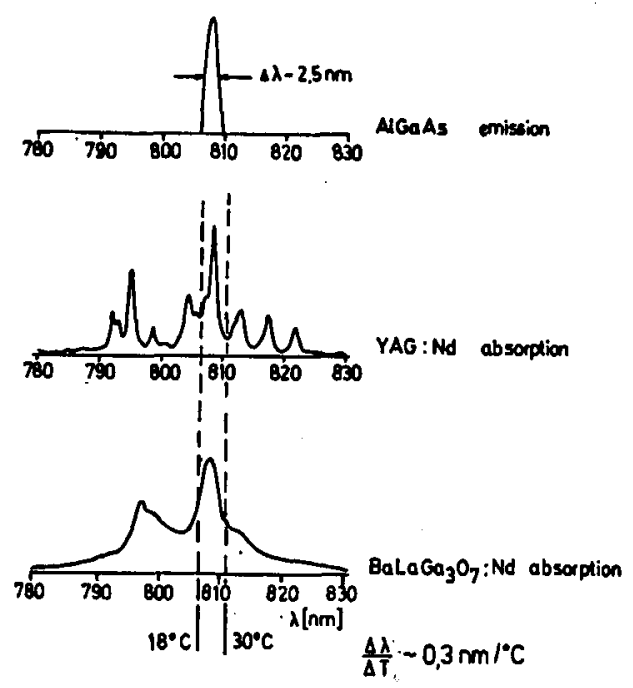

Fig. 2. Emission band of a commercial AlGaAs laser diode and absorption bands of YAG:Nd and $\mathrm{BaLaGa}_{3} \mathrm{O}_{7}: \mathrm{Nd}$.

tially one or more cations by cations with different charges. In this way a number of so-called substituted garnets has been obtained. The second way is to synthesize compounds with inherently disordered structure. Up to now, several compounds has been obtained and studied as potential laser materials. Chemical composition of the crystals of both the groups are listed below.

Substituted garnets: $\mathrm{Ba}_{0.25} \mathrm{Mg}_{2.75} \mathrm{Y}_{2} \mathrm{Ge}_{3} \mathrm{O}_{12} \quad$ [4], GGG(Ca,Zr), $\mathrm{GGG}(\mathrm{Ca}, \mathrm{Mg}, \mathrm{Zr})[2,3], \mathrm{Ca}_{3}\left(\mathrm{Nb}, \mathrm{Ga}_{2}\right) \mathrm{Ga}_{3} \mathrm{O}_{12}[5]$.

Inherently disordered structures: $7 \mathrm{Ln}_{2} \mathrm{O}_{3} \cdot 9 \mathrm{SiO}_{2}$ where $\mathrm{Ln}=\mathrm{La}$ or $\mathrm{Gd}[6]$, $\mathrm{Ca}_{2} \mathrm{Ga}_{2} \mathrm{SiO}_{7}$ [7], $\mathrm{LaSr}_{2} \mathrm{Ga}_{11} \mathrm{O}_{20}$ [8], $\mathrm{Sr}_{1-x} \mathrm{Nd}_{x} \mathrm{Mg}_{x} \mathrm{Al}_{12-x} \mathrm{O}_{19}$ [9], $\mathrm{BaLaGa}_{3} \mathrm{O}_{7}$ [10], $\mathrm{SrLaGa}_{3} \mathrm{O}_{7}$ [11], $\mathrm{CaNdAlO}_{4}$ [12], $\mathrm{SrLaAlO}_{4}$.

Let us have a look at the structural features which give rise to inhomogeneous broadening. We will consider first the compounds $\mathrm{ABC}_{3} \mathrm{O}_{7}$, where $\mathrm{A}$ stands for $\mathrm{Ca}, \mathrm{Sr}, \mathrm{Ba}, \mathrm{B}$ stands for $\mathrm{Y}$ or rare earth from $\mathrm{La}$ to $\mathrm{Gd}$ and $\mathrm{C}$ stands for $\mathrm{Al}$ or $\mathrm{Ga}$. These compounds form tetragonal crystals belonging to the space group $P 42_{1} m$. Lattice constants are $a=8.05 \AA$ and $c=5.16 \AA$ for $\mathrm{BaLaGa}_{3} \mathrm{O}_{7}$ and they are very similar for other compositions.

The primitive cell and the $c$-axis projection of the $\mathrm{ABC}_{3} \mathrm{O}_{7}$ structure is shown in Fig. 3. The crystal structure is built up from the layers of $\mathrm{CO}_{4}$ polyhedra. There are two types of tetragonal sites $T_{1}, T_{2}$ and $T_{3}-T_{6}$. Between the layers the $A$ and $B$ cations are located in sites of very distorted square antiprism configuration. In Fig. 3 the shaded areas are four edge faces of square antiprism facing the observer. There are similar faces at the other side of the layer. Above the layer, at the top of shaded area, the divalent $A$ cations and trivalent $B$ cations are randomly distributed in the sites of $C_{s}$ symmetry. Disparity of size and of charge 

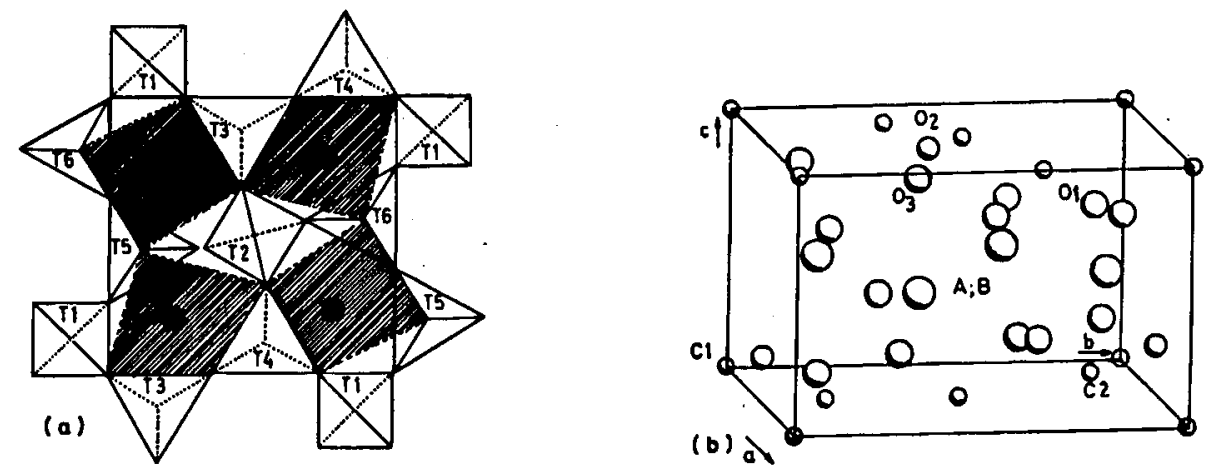

Fig. 3. $C$-axis projection (a) and primitive cell (b) of $\mathrm{ABC}_{3} \mathrm{O}_{7}$ crystals.

of ions in neighbouring sites gives rise to random distortion of crystal field acting on activating neodymium ions which substitute partially $\mathrm{La}$ in the structure. The primitive cell of this structure contains two formula units and the minimal distance between two neodymium ions is inferior to $4 \AA$, thus the ion-ion interactions are expected to be considerable.

Another example of disordered structure is formed by $\mathrm{ABCO}_{4}$ compounds. In this general formula $\mathrm{A}$ stands for $\mathrm{Ca}, \mathrm{Sr}, \mathrm{Ba}, \mathrm{B}$ stands for $\mathrm{Y}$ or for rare earth ions and $\mathrm{C}$ stands for $\mathrm{Al}, \mathrm{Ga}$. The crystals are also tetragonal but they belong to the space group $14 m m m$. Lattice parameters for $\mathrm{CaNdAlO}_{4}$ are $a=3.68 \AA$ and $c=12.11 \AA$ and they do not differ significantly for other compositions. In Fig. 4 we present the positions of ions and the polyhedra forming the $\mathrm{ABCO}_{4}$ structure. The crystals are built up from $\mathrm{AlO}_{6}$ layers formed in the $a b$ plane. Between the layers the divalent $A$ cations and trivalent $B$ cations are randomly distributed in the sites of $C_{4 v}$ symmetry. Minimal distance between neodymium ions is inferior to $4 \AA$ like in the former structure.

In both the groups of compounds the structure is very sensitive to the size of ions $A$ and $B$. The lower the difference between ionic radii of $A$ and $B$, the more stable is the structure. As was said, the strong inhomogeneous broadening of absorption bands is advantageous for optical pumping. There are however other consequences which are not necessarily favourable for laser application.

Let us try to assess the laser performance of disordered crystals. In practical application the low threshold pumping power will be requested. Threshold pumping power is a function of several factors and may be expressed by the following formula [1]:

$$
P_{\mathrm{th}}=\frac{L h v_{\mathrm{p}} V_{\mathrm{eff}}}{2 l \sigma \tau \eta_{\mathrm{p}} f_{\mathrm{B}}}
$$

where $L$ is the round trip cavity loss, $l$ is the cavity length, $V_{\text {eff }}$ is the effective mode volume, $\eta_{\mathrm{p}}$ is the absorption efficiency, $\sigma$ is the laser emission cross-section, $\tau$ is the fluorescence lifetime, $f_{\mathrm{B}}$ is the fractional Boltzmann distribution. In the formula the effective mode volume and the cavity length are the design parameters 

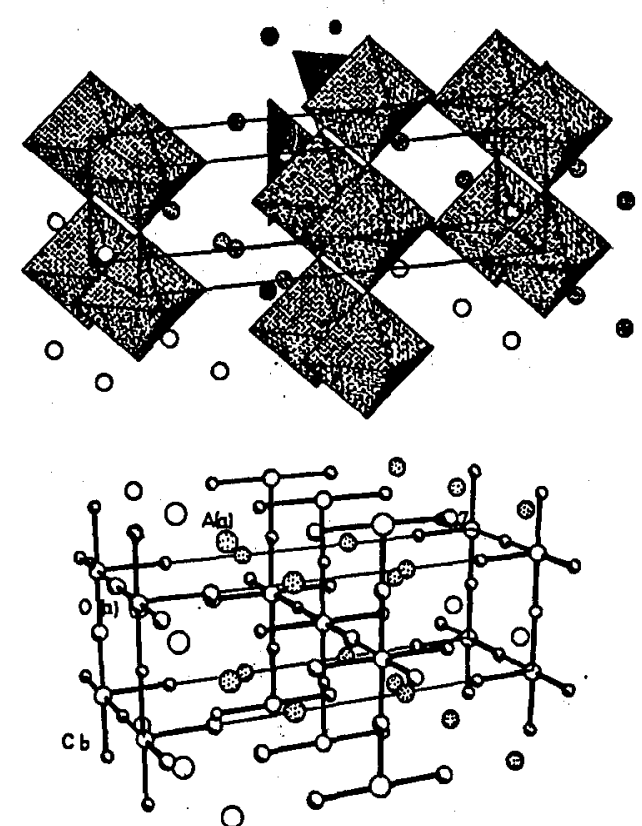

Fig. 4. The position of ions (lower part) and polyhedra (upper part) forming the $\mathrm{ABCO}_{4}$ structure.

whereas the lifetime of upper laser level, stimulated emission cross-section and the Boltzmann distribution factor are material parameters. The round trip loss $L$ accounts for both the losses in active material and the output mirror transmission. Absorption efficiency takes into account the fraction of laser diode power absorbed and the loss due to photon decrement and may be approximated by the following formula [14]:

$$
\eta_{\mathrm{p}}=\frac{\int_{\lambda_{1}}^{\lambda_{2}} \int_{0}^{\frac{\pi}{2}} \int_{0}^{\frac{\pi}{2}} \frac{\lambda}{\lambda_{1}} P(\lambda) F(\Theta, \phi)[1-\exp (-\beta(\lambda) l)] \sin \Theta \mathrm{d} \Theta \mathrm{d} \phi \mathrm{d} \lambda}{\int_{\lambda_{1}}^{\lambda_{2}} \int_{0}^{\frac{\pi}{2}} \int_{0}^{\frac{\pi}{2}} P(\lambda) F(\Theta, \phi) \sin \Theta \mathrm{d} \Theta \mathrm{d} \phi \mathrm{d} \lambda},
$$

where $P(\lambda)$ is the spectral distribution of laser diode radiation, $F(\Theta, \phi)$ is the angular distribution of laser diode radiation, $\beta(\lambda)$ is absorption coefficient, $\lambda_{1}$ is the laser wavelength. This parameter may be adjusted by appropriate doping, provided the host crystal accepts desirable amount of neodymium ions. Since the disordered crystals considered here may be heavily doped, the absorption efficiency is not a limiting factor. However, two other material parameters, namely the lifetime of upper laser level and the stimulated emission cross-section cannot be adjusted. Stimulated emission cross-section is related to spectral properties of laser material according to the formula

$$
\sigma=\frac{\lambda^{2} W_{\mathbf{r}}}{8 \pi c n^{2} \Delta \nu_{\mathrm{efr}}}
$$


where $\lambda$ denotes the wavelength of laser transition, $W_{\mathrm{r}}$ is the rate of spontaneous radiative transition from upper laser level to lower laser level, $n$ is the index of refraction, $\Delta \nu_{\text {eff }}$ is an effective bandwidth of luminescence associated with laser transition. Two methods are commonly used for the calculation of this parameter. The first one makes use of relation (3) and the rate of radiative transition is determined on the basis of the Judd-Ofelt theory. The second method is based on measurements of threshold pumping power for different output mirror transmissions. In this case the relation (1) is used. Both the methods rely on approximations and the errors of calculation may exceed even $50 \%$ and this should be taken into account when any comparison is made. Stimulation emission cross-section is inversely proportional to bandwidth of transitions, thus disordered crystals will not show high values of this parameter.

For comparison in Fig. 5 the luminescence bands corresponding to laser transition in different crystals are presented. Bandwidths of the ${ }^{4} F_{3 / 2} \rightarrow{ }^{4} I_{11 / 2}$ transi-

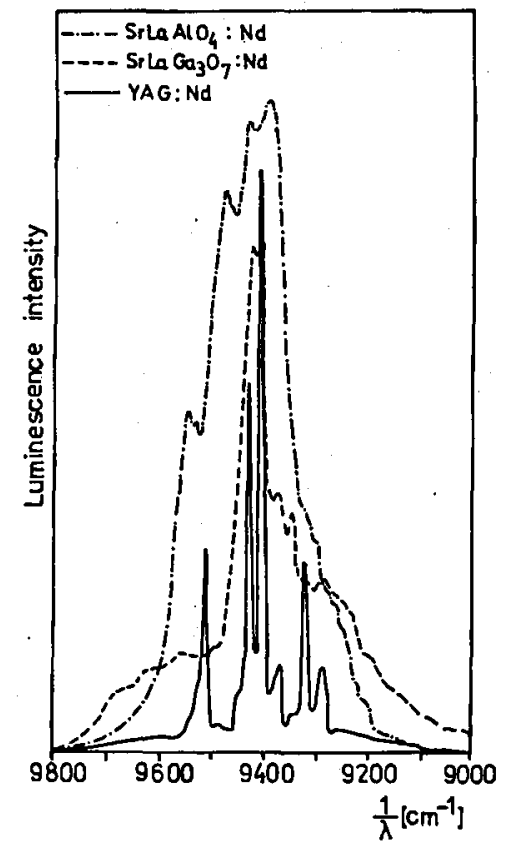

Fig. 5. The luminescence bands corresponding to laser transition in YAG:Nd, $\mathrm{SrLaAlO}_{4}: \mathrm{Nd}, \mathrm{SrLaGa}_{3} \mathrm{O}_{7}: \mathrm{Nd}$.

tion of $\mathrm{Nd}^{3+}$ are about $6 \mathrm{~cm}^{-1}$ in YAG, $160 \mathrm{~cm}^{-1}$ in $\mathrm{BaLaGa}_{3} \mathrm{O}_{7}$ and about 220 $\mathrm{cm}^{-1}$ in $\mathrm{SrLaAlO}_{4}$. Lifetime of upper laser level influences the threshold value but also the amount of energy stored in the upper laser level during pulsed operation. Assuming a square pumping pulse, the storage efficiency, that is the fraction of stored energy which is available in the time of $Q$-switching may be approximated 
as

$$
\eta_{\mathbf{s}}=\frac{\tau_{2}}{\tau_{l}}\left[1-\exp \left(\tau_{l} / \tau_{2}\right)\right],
$$

where $\tau_{2}$ is the lifetime of upper laser level, $\tau_{1}$ is the length of pumping pulse. Long lifetime is thus advantageous in pulsed operation.

Now, we can compare the available data relevant for laser performance of some disordered crystals. In Table the lifetimes $\tau$, stimulated emission cross-sections $\sigma$ and the products $\sigma \tau$ are presented.

TABLE

Luminescence lifetimes $\tau$ and laser emission cross-sections $\sigma$ for $\mathrm{Nd}^{3+}$ in disordered crystals.

\begin{tabular}{l|c|c|c|c}
\hline Host crystal & $\tau[\mu \mathrm{s}]$ & $\begin{array}{c}\sigma \times 10^{20} \\
{\left[\mathrm{~cm}^{2}\right]}\end{array}$ & $\begin{array}{c}\sigma \tau \times 10^{23} \\
{\left[\mathrm{~cm}^{2} \mathrm{~s}\right]}\end{array}$ & Ref. No. \\
\hline $\mathrm{GGG}(\mathrm{Ca}, \mathrm{Zr})$ & 230 & 7.0 & 1.61 & {$[15]$} \\
$\mathrm{GGG}(\mathrm{Ca}, \mathrm{Mg}, \mathrm{Zr})$ & 260 & 4.9 & 1.27 & {$[15]$} \\
$\mathrm{Sr}_{1-x} \mathrm{Nd}_{x} \mathrm{Mg}_{x} \mathrm{Al}_{12-x} \mathrm{O}_{19}$ & 230 & 4.8 & 1.10 & {$[15]$} \\
$\mathrm{Ca}_{2} \mathrm{Ga}_{2} \mathrm{SiO}_{7}$ & 280 & 5.3 & 1.48 & {$[13]$} \\
$\mathrm{LaSr}_{2} \mathrm{Ga}_{11} \mathrm{O}_{20}$ & 220 & 5.5 & 1.21 & {$[13]$} \\
$\mathrm{BaLaGa}_{3} \mathrm{O}_{7}$ & 300 & 5.0 & 1.50 & {$[16]$} \\
$\mathrm{SrLaGa}_{3} \mathrm{O}_{7}$ & 300 & 6.01 & 1.79 & {$[16]$} \\
$\mathrm{SrLaAlO}_{4}$ & 140 & 5.9 & 0.83 & this work
\end{tabular}

It can be seen that the values of stimulated emission cross-section are of the same order. The products show more significant differences, but still they are roughly one order of magnitude lower than that for YAG:Nd. Thus, the gain in overall laser efficiency resulting from the elimination of the cooling device is counterbalanced to some extent by higher threshold pumping powers. It seems that disordered laser crystals are particularly suited for high power and very short pulse operation where the effects of spontaneous amplified emission limit the efficiency of high gain ordered crystals like YAG:Nd. Because of the similarity of values in Table, the choice of a crystal for practical application will rely on no spectral parameters, such as thermal conductivity, attainable optical quality or the cost of production.

The work was supported by the Committee for Scientific Research under grant No. 203589101.

\section{References}

[1] R.A. Fields, M. Birnbaum, C.L. Fincher, Appl. Phys. Lett. 51, 1885 (1987).

[2] L. Zhang, L. Liu, M. Liu, C. Lin, J. Cryst. Growth 80, 257 (1987).

[3] L.H. Zhang, Prog. Cryst. Growth Charact. 11, 283 (1985). 
[4] I.E. Miller, E.J. Sharp, D.J. Horowitz, J. Appl. Phys. 43, 462 (1972).

[5] N.A. Eskov, M.D. Faerman, N.A. Surova, I.V. Ostrovskii, N.A. Groshenko, Ukr. Khim. Zh. 51, 457 (1985).

[6] A.M. Korovkin, T.I. Merkulyaeva, L.G. Morozova, A. Peschanskaya, M.V. Petrov, I.R. Savinova, Opt. Spectrosc. 58, 778 (1985).

[7] A.A. Kaminskii, E.L. Belokoneva, B.V. Mill, S.E. Sarkisov, K. Kurbanov, Phys. Status. Solidi A 97, 279 (1986).

[8] A.A. Kaminskii, B.V. Mill, E.L. Belokoneva, A.V. Butashin, S.E. Sarkisov, K. Kurbanov, G.G. Khodzhabagyan, Izv. Akad. Nauk SSSR Neorg. Mater. 22, 1869 (1986).

[9] S. Alablànche, R. Collongue, M. Leduc, A. Minvielle, J. Thiery, D. Vivien, in: Laser M2P. Conf., Grenoble (France) 1991, p. D4.

[10] W. Ryba-Romanowski, M.U. Gutowska, L. Piekarczyk, M. Berkowski, Z. Mazurak, B. Jeżowska-Trzebiatowska, J. Lumin. 38, 369 (1987).

[11] W. Ryba-Romanowski, S. Gołąb, M. Berkowski, Proc. SPIE, Laser Technology II 1391, 2 (1990).

[12] W. Ryba-Romanowski, S. Gołąb, J. Hanuza, M. Mączka, A. Pietraszko, M. Berkowski, A. Pajączkowska, J. Phys. Chem. Solids 52, 1043 (1991).

[13] A.A. Kaminskii, H.R. Verdun, B.V. Mill, Phys. Status Solidi A 129, K125 (1992).

[14] N.P. Barnes, M.E. Storm, P.L. Cross, M.L. Skolant, IEEE J. Quantum Electron. 26, 558 (1990).

[15] N. Mermilliod, R. Romero, I. Chartier, C. Garapon, R. Moncorge, IEEE J. Quantum Electron. 28, 1179 (1992).

[16] W. Ryba-Romanowski, S. Gołąb, G. Dominiak-Dzik, M. Berkowski, Mater. Sci. Eng. B 15, 217 (1992). 